\title{
IMPROVEMENT AND MAINTENANCE \\ OF THE EXTRAGALACTIC REFERENCE FRAME
}

\author{
E.F. ARIAS and A.M. GONTIER
}

\begin{abstract}
Very Long Baseline Interferometry (VLBI) is at present the most powerful technique to construct the best approximation to an inertial reference frame. After more than a decade of VLBI observations several hundreds of extragalactic objects have positions known within \pm 0.0003 ". Since 1988 the International Earth Rotation Service (IERS) elaborates a global extragalactic celestial reference frame (IERS Celestial Reference Frame, ICRF) that is tied to the international terrestrial reference frame through the high precision monitoring of the Earth's rotation. The direction of the ICRF axes relative to the IAU definitions are known within better than $\pm 0.001 "$ for the polar axis and \pm 0.003 " for the origin of right ascensions. The FK5 axes are consistent with the ICRF ones within their uncertainties (0.050"-0.100"). The maintenance of this high accuracy extragalactic frame will be necessary for the long term programs, such as the future monitoring of the tie of the Hipparcos galactic frame as well as of the dynamical planetary frame (millisecond pulsars, lunar laser ranging).
\end{abstract}

